\title{
Development of a toolkit for early identification of cauda equina syndrome
}

\author{
Sue Greenhalgh ${ }^{1}$, Carole Truman $^{2}$, Valerie Webster ${ }^{3}$ and James Selfe ${ }^{4}$ \\ ${ }^{1}$ Consultant Physiotherapist, Elective Orthopaedic Department, Bolton One, Moor Lane, Bolton, BL35BN, UK \\ ${ }^{2}$ Independent Research Consultant \\ ${ }^{3}$ Executive Dean and Pro Vice-Chancellor of the School of Health and Life Sciences, Glasgow Caledonian University, \\ Cowcaddens Road, Glasgow G4 0BA, Scotland, UK \\ ${ }^{4}$ Department of Health Professions, Faculty of Health, Psychology and Social Care, Manchester Metropolitan University \\ Brooks, Building Birley, Manchester, M15 6GX, UK
}

\begin{abstract}
Aim: To develop a simple cauda equina syndrome (CES) toolkit to facilitate the subjective examination of low back pain patients potentially at risk of CES. To undertake preliminary validation of the content of the toolkit. Background: CES is a rare condition which can be very challenging to identify in a generalist medical setting. Method: A three phase iterative design with two stake holder groups; extended scope practitioners experienced in managing CES patients and CES sufferers. Toolkit development: Synthesis of existing CES literature with CES patient data generated from in depth interviews. Toolkit validation: Content validation of the draft toolkit with CES patients. Toolkit validation: Content validation of the draft toolkit with extended scope physiotherapists. Findings: A three arm toolkit has been developed for use with patients considered by the clinician as at risk of developing CES (eg, worsening low back pain with symptoms/signs of progressive sensory-motor deficit in the lower limbs); patient expertise, clinical expertise, research and pathways. Uniquely, the toolkit drew upon the lived experiences of patients suffering from CES to inform the content.
\end{abstract}

Key words: cauda equina syndrome; development; toolkit

Received 15 June 2015; revised 31 July 2015; accepted 16 February 2016;

first published online 21 April 2016

\section{Background/introduction}

Cauda equina syndrome (CES) is a rare condition affecting $2 \%$ of all herniated discs; it occurs as a consequence of neural compression, leading to loss of function of two or more of the 18 nerve roots which comprise the cauda equina (Woolsley and Martin, 2003). Neural compression is usually due to intervertebral disc herniation or prolapse. CES is considered a potential emergency within orthopaedics (Fraser et al., 2009; Mestrum et al., 2009), however, the exact timing of surgical decompression remains controversial (Chau et al., 2014).

Correspondence to: Dr Sue Greenhalgh, Consultant Physiotherapist, Elective Orthopaedic Department, Bolton One, Moor Lane, Bolton BL35BN, UK. Email: Susan.greenhalgh@ boltonft.nhs

(C) Cambridge University Press 2016
Early diagnosis and surgical decompression are essential. Jalloh and Minhas (2007) and Gleave and Mac Farlane (2002) in their seminal paper suggest that spinal surgery within $48 \mathrm{~h}$ of an individual developing sphincter dysfunction will optimise postoperative recovery. If left untreated, CES can lead to permanent loss of bowel and bladder control, sexual dysfunction or even paralysis (Markham, 2004).

Patients suffering from CES often present to a variety of non-specialist front-line services such as A\&E and physiotherapy; the identification of CES in a generalist medical setting can be very challenging. It is estimated that a GP, a generalist in relation to CES, will witness the onset of CES in only one patient in their entire career (Underwood, 2009). This militates against experiential pattern recognition and 'routinisation' (Eraut, 2000) which may contribute to delays in diagnosis and onward 
appropriate referral. In a retrospective evaluation of 753 consecutive LBP patients in Oxford, 28\% reported altered bladder and bowel function and only one of these patients had a radiologically confirmed CES that was managed by emergency surgery (Buchanan, 2013). Due to the serious consequences of and suffering experienced by people with the condition; CES has become highly litigious (Markham, 2004) with an average payment of $£ 336000$ (Fairbank, 2014). The NHS has paid out circa. $£ 44 \mathrm{~m}$ in the 10 years up to 2013, for CES related claims (NHS Litigation Authority, 2013).

Despite the rarity of true CES, frontline clinicians should maintain a high index of suspicion to avoid diagnostic delay. While clinicians should routinely check for symptoms related to the Cauda Equina in patients presenting with back problems, any combination of bladder, bowel and sexual dysfunction should not be ignored (Selius and Subedi, 2008). Many concomitant issues can cause symptoms which masquerade as CES, such as opioid analgesics, for example Codeine or Tramadol are well known for commonly causing constipation. CES patients often concurrently experience severe unremitting pain which from the patient's perspective is the most significant symptom in the early stages (Greenhalgh et al., 2014). Pain levels can complicate the clinical reasoning process; therefore, clinicians need to draw on a variety of clinical decision making strategies (André et al., 2012). Good patient/clinician communication is crucial; with clinicians having expert, tailored questioning skills to gain key information about CES symptoms from the subjective examination. Importantly, pain distracts attention away from possible CES specific symptoms, especially when subtle and vague (Bin et al., 2009). In addition, pain can also contribute to symptoms of retention (Korse et al., 2013).

This paper is part of a programme of work investigating Patient Information Supplements for Cauda Equina Syndrome and it describes the development of a clinical toolkit designed to enhance the early identification of CES. Toolkits are described by Monroe (2000) as a collection of educational information and resources targeting one issue or one audience. Widely used in health care settings, toolkits are often used as a resource when considering more efficient ways of delivering health care systems or ways to improve clinical diagnosis. For example, in response to repeated studies identifying poor outcomes for cancer patients in the
United Kingdom, Mitchell et al. (2012) developed a Toolkit based on the experiences of general practitioners participating in initiatives for the early identification of cancer. Clinical Toolkits often reflect or are grounded in the perspectives of multiple stakeholder groups including patients or service users. For example, Vrkljan et al. (2010) developed a Toolkit outlining strategies to support safe mobility for people with arthritis drawing on the experience of both patients and clinicians.

An approach similar to Vrkljan et al. (2010) was adopted by undertaking a developmental study drawing on two different stakeholder group perspectives, sufferers of CES and extended scope physiotherapy practitioners (ESPs). Throughout this paper patient pseudonyms have been utilised where direct quotes have been provided. This builds on previous qualitative interview work undertaken with sufferers of CES (Greenhalgh et al., 2015). The data gained through these aforementioned patient interviews generated key issues that were perceived to lead to diagnostic delays. These key issues informed the following research questions

- 'What are the key CES symptoms that patients should look out for and clinicians should specifically ask questions about?'

- 'If the patient develops any of these symptoms what action should they take and when?'

To answer these questions a three part developmental study was conducted with the following objectives.

\section{Objectives}

- To develop a simple CES toolkit to facilitate the subjective examination of low back pain patients potentially at risk of CES.

- To undertake preliminary validation of the content of the toolkit.

\section{Methods}

- Synthesis of existing CES literature with CES patient data generated from in depth interviews (Greenhalgh et al., 2015). Setting academic institution

- Content validation of the draft toolkit with CES patients. Setting patients' homes via telephone 
- Content validation of the draft toolkit with extended scope physiotherapists. Setting Primary Care Health Centre

\section{Inclusion criteria}

- Patients who had previously been involved in the interview study (Greenhalgh et al., 2015).

- ESP physiotherapists with experience of dealing with CES patients from 2 NHS trusts.

\section{Exclusion criteria}

In order to reduce social desirability bias physiotherapists working in the same NHS Trust as the lead author were excluded.

\section{Ethical approval}

Ethical approval was granted by NRES committee North West - Liverpool Central (12/NW/0529).

\section{Toolkit development: synthesis of existing CES literature with CES patient data generated from in depth interviews}

As described in full, in Greenhalgh et al. (2015), seven themes emerged from the in depth analysis and exploration of data generated from interviews with CES patients.

- Pain

- Impact on life

- Common symptoms, varying chronology

- Sense of change/seriousness

- Contact with health professionals

- Carers experience

- Suggestions to aid early diagnosis

On exploration of the raw data supporting the Contact with Health Professionals theme, it was clear that effective clinician patient communication was a significant issue where improvements needed to be made.

'They really do need to listen to you' (Mrs Brown)

'...nobody's taking any notice of me' (Mrs Red)

'I've never been asked about the sexual function side of things' (Mrs White).

Also of interest was that in line with INVOLVE Guidelines (NIHR, 2014) the patients use of particular language had emerged as especially important. What was clear was that patients used a very explicit language in their description of CES symptoms For example

'I wasn't weeing properly, it was just spurting and spraying so I knew that wasn't right' (Mrs Brown)

and

'If I had been told numbness around back passage or genitals...everyone I saw who was medically trained called it saddle numbness' (Mr Black).

It was vital that this type of patient centred language informed the development of the toolkit to assist clinicians identify CES. Following the thematic analysis of the patient's interviews a critical review of key published material was undertaken. The following documents were reviewed:

- CES UK Charity patient guidance leaflet and clinician leaflet (CES UK Charity, 2014)

- UK Case book (Anthony, 2003)

- Developing an early alert system for metastatic spinal cord compression (Turnpenney et al., 2013)

- Gloucestershire physiotherapy department CES protocol (GHNHSFT, 2013).

Importantly it emerged that there was a dichotomy between the language used by the patients to describe a wide scope of CES symptoms and the language of professionals that generally referred to a restricted scope of CES symptoms.

'The GP said it's serious if you are incontinent... I had to force myself to go but I was still going to the toilet so I did not see a problem' (Mr Black).

Following synthesis of the existing CES literature with the CES patient data, a toolkit with three arms was developed (Figure 1). The three arms are; Patient Expertise, Clinical Expertise, Research and Pathways. The toolkit also contains a clinical cue card (Figure 2) and a credit card sized patient information card (Figure 3).

\section{Content validation of the draft toolkit with CES patients}

All participants in the interviews were sent a draft toolkit through the post, with a letter of 
explanation and an invitation to take part in a telephone interview. The purpose of the telephone interview was to elicit comments on the draft CES toolkit and its content. Field notes were taken though out the telephone calls and suggested changes made to the toolkit. The participant validation (led by S.G.) provided confirmation that the toolkit reflected participants own language and established that the toolkit appeared fit for purpose.

\section{Content validation of the draft toolkit with extended scope physiotherapists}

Following the patient participant validation the toolkit was also validated from a clinician's perspective. This process was led by J.S. and involved seven Spinal Extended Scope Practitioner's experienced in the management of patient's with CES from two NHS Health Trusts within the North West of England. The process began with a brief presentation of the draft Toolkit validated by the participants who were all CES sufferers. The presentation also included a full explanation of the process of development of the Toolkit. A focus group exploring aspects of the draft toolkit was then conducted with the Extended Scope Practitioners. Data generated during the focus group led to the final amendments to the draft CES toolkit being made.

\section{Results}

\section{Toolkit: patient expertise (Figure1)}

Patients have first-hand knowledge of their own symptoms hour by hour. As timing to surgical opinion is paramount, patients need to be empowered with detailed information of what symptoms to look out for and precisely what to do about health seeking, that is what time frame and what action.

'I did not think it [saddle numbness] was significant......If I had known how serious this was...if I had known that 12 hours can make a hell of a difference.... I only got the literature after the surgery' (Mr Black).

In addition patients expressed the value of having literature to take to an emergency appointment to assist in their explanation of their concerns to the medical practitioner. Patient participants reported that they felt it would help them in an emergency clinical situation;

'to stand their ground' (Mrs White).

In addition, sufferers could explain their important emerging symptoms in a more clearly articulated manner that clinicians would understand. For example, Mrs White presented to A\&E with CES but she found her saddle numbness and

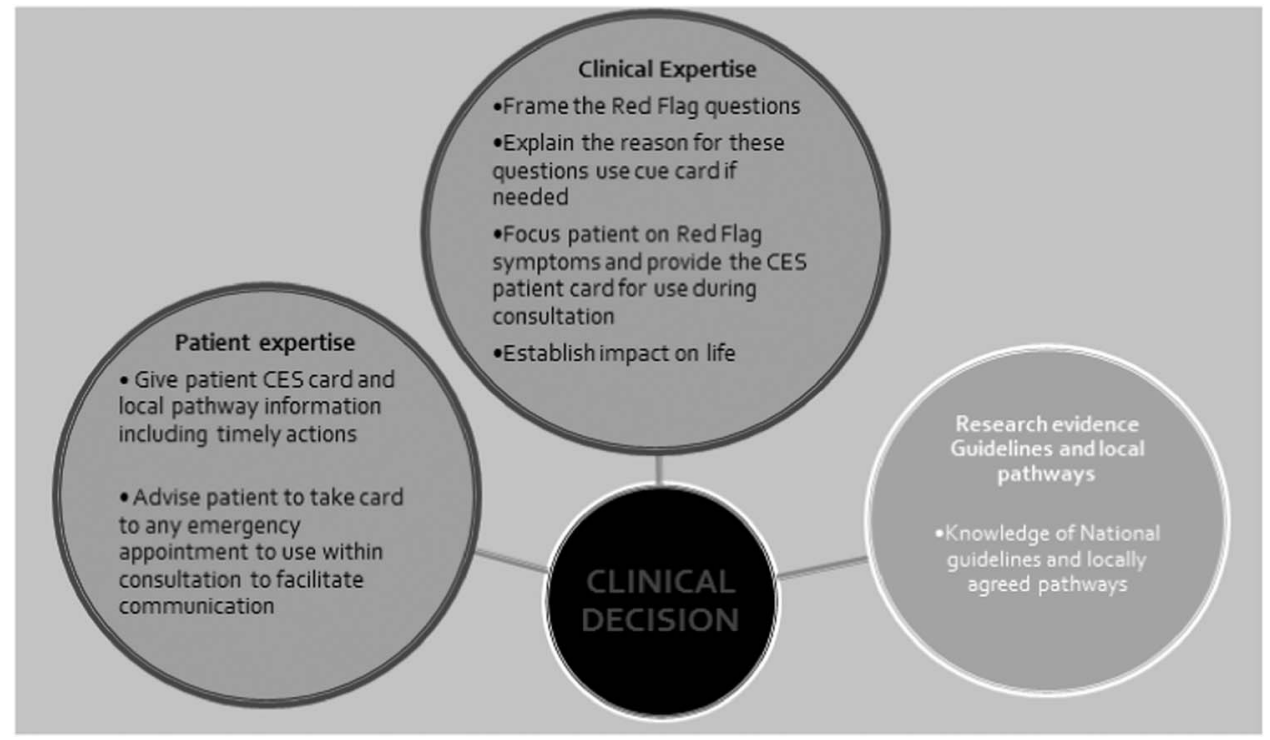

Figure 1 CES toolkit. CES = cauda equina syndrome

Primary Health Care Research \& Development 2016; 17: 559-567 
- Loss of feeling/pins and needles between your inner thighs or genitals

- Numbness in or around your back passage or buttocks

- $\quad$ Altered feeling when using toilet paper to wipe yourself

- Increasing difficulty when you try to urinate

- Increasing difficulty when you try to stop or control your flow of urine

- Loss of sensation when you pass urine

- Leaking urine or recent need to use pads

- Not knowing when your bladder is either full or empty

- Inability to stop a bowel movement or leaking

- Loss of sensation when you pass a bowel motion

- Change in ability to achieve an erection or ejaculate

- Loss of sensation in genitals during sexual intercourse

Any combination of the above warning signs could be symptoms of Cauda Equina Syndrome. Seek Emergency medical help within 12 to 24 hours

Figure 2 Cauda equina syndrome warning signs cue card for clinicians

\section{Gauda Equina Syndrome Warning Signs}

- Loss of feeling/pins and needles between your inner thighs or genitals

- Numbness in or around your back passage or buttocks

- Altered feeling when using toilet paper to wipe yourself

- Changes when you try to urinate

- Changes when you try to stop or control your flow of urine

- Loss of sensation when you pass urine

- Leaking urine or recent need to use pads

- Not knowing when your bladder is either full or empty

- Inability to stop a bowel movement or leaking

- Loss of sensation when you pass a bowel motion

- Change in ability to achieve an erection or ejaculate

- Loss of sensation in genitals during sexual intercourse

\begin{tabular}{|} 
Any combination or \\
number of these \\
warning signs \\
could be symptoms \\
of Cauda Equina \\
Syndrome. \\
Seek emergency \\
medical help within \\
12 to 24 hours \\
\end{tabular}

Figure 3 Patient credit card

urinary symptoms difficult to explain and was sent away with reassurance.

'We came away [from A\&E] because you trust what they say' (Mrs White).
Mrs White did have CES at that presentation and eventually underwent spinal surgery.

\section{Toolkit: clinical expertise (Figure 1)}

Communication of the gravity of CES is a key component of the consultation with patients at risk of CES. Patients described not recognising the importance of Red Flag questions. Clinicians need to explain the condition using non-medical lay terms, with emphasis on the seriousness and importance of the timeframe. Using language understood by both patients and clinicians is vital when describing symptoms to look out for. A clinical cue card (Figure 2) was developed as part of this arm of the toolkit to assist clinicians engage in meaningful dialogue with patients; it lists 12 items in bullet point format focussing on the explicit bladder, bowel and sexual dysfunction features of the syndrome. The clinical cue card was initially suggested as potentially useful by patients.

Primary Health Care Research \& Development 2016; 17: 559-567 
In clinical practice this cue card can be adapted into different formats, for example on an A4 laminated paper or on a computer screen etc. The language used in the version presented in Figure 2 is the final version developed following the two part validation process. This same information was replicated on a small credit card for the patient to take away and use in any future CES emergency situation enabling explanation of all symptoms fully (Figure 3 ). Finally critical information of what actions to take and in what time frame must be clearly articulated to the patient.

'I think there should be something about how urgent it can be' (Mr Black).

\section{Toolkit: research and pathways (Figure 1)}

From the outset it was obvious that any new CES toolkit would need to incorporate the most up to date research and needed to be consistent with defined local clinical pathways. However despite local pathways being in place for the patient participants, timely surgical opinion still emerged as being inconsistent. This triggered the notion that a CES toolkit may be a useful aid to clinical practice.

\section{Discussion}

One of the main drivers for this research was that despite up to date evidence and appropriate clinical pathways being in place timing to surgical opinion was not always consistent. In the past ten years the emergency surgical window has been regularly reported as small, that is $48 \mathrm{~h}$ (Chau et al., 2014). In order to be clinically useful the toolkit (Figure 1) needed to be concise, evidence based and facilitate meaningful two way dialogue between patient and clinician. In particular it needed to provide patients with knowledge about CES and clinicians with a language that patients could understand (CES UK Charity, 2014; NIHR 2014). Uniquely the toolkit drew upon the lived experiences of patients suffering from CES to inform the content. In line with INVOLVE Guidelines (NIHR, 2014) patients were engaged as equal partners in the development of the toolkit as they are experts in their own signs and symptoms associated with CES. In addition the patient participants ensured the language and content of information was appropriate and accessible (NIHR, 2014).

Through this study it emerged that in order to identify CES patients early in the disease process to facilitate timely surgical opinion one of the key issues was the use of language that reflected the patient's own voice. The patient participants highlighted the need for clinicians to use language that they could readily understand during a clinical consultation and they suggested the development of a CES cue card for clinicians (Figure 2). They also suggested that the clinical cue card and patient credit card (Figure 3) map against each other using the same questions. With this in mind an iterative validation process took place. Current evidence suggests that progressing motor \& sensory deficits are important, however, Sun et al. (2014) highlights the importance of bladder, bowel and sexual function symptoms in the timely diagnosis of CES. The preservation of these normal functions at the time of diagnosis is directly related to outcome. Therefore the content of the patient and clinician cards focused on these issues.

The research team engaged in negotiation around the specific language, words and inferences that appeared on the final versions of the clinical cue card (Figure 2) and patient credit card (Figure 3). For example 'Recent onset of sexual dysfunction' had little meaning to patients. As an alternative the patients highlighted the need for much more specific language around sexual function rather than general questions suggestive of sexual activity as many were not actually sexually active for a variety of reasons some unconnected to the physiological changes associated with CES. For example CES sufferer Mrs. White stated 'I've never been asked about sexual function side of things but my husband doesn't have that anymore so it doesn't happen anyway'. Therefore 'Change in ability to achieve an erection or ejaculate; and 'Loss of sensation in genitals during sexual intercourse' was chosen to be included in the final version of the toolkit instead of 'Recent onset of sexual dysfunction'. Patient participants also highlighted the need for the timing issues associated with seeking medical help relative to the surgical window of opportunity to be emphasised. This underpins the empirical evidence of Gleave and MacFarlane (2002) and Jalloh and Minhas (2007) who identified that patients with CES are managed in a timely manner as early surgery for 
confirmed cases may avoid serious complications. Therefore, in order to attain the $48 \mathrm{~h}$ surgical window, the phrase on the patient card 'seeks medical help within 12 to 24 hours' was changed to 'seek emergency medical help within 12 to 24 hours'.

'Urgent to me doesn't mean right away....it's the name of an appointment

\section{...Emergency means right away' (Mr Black)}

'If I had been given that piece of information

I would have been in 12 hours earlier' (Mr Black).

The clinicians felt that the toolkit and the two cards were useful, would enhance clinical decision making and had the potential to improve patient care. They suggested consistency in the use of the word 'change'; as a consequence two of the items that started with the word 'difficulty' were amended. From a very practical perspective they also suggested that the patient card (Figure 3) be constructed with a fold out leaf on which specific local emergency actions could be documented, for example a named persons emergency contact details. Interestingly the therapists expressed surprise at the explicit nature of the language that the patient participants had preferred to use on the clinicians cue card (Figure 2) and the patients credit card (Figure 3 ). This appears to highlight the gap between what clinicians perceive they are asking and what patients perceive is being asked. This reinforces the importance of a shared language that both parties understand (Anthony 2003; NIHR, 2014).

Anthony (2003) highlights that the patients experience of CES symptoms can be difficult for them to recognise and or articulate. These issues are exemplified by the experience described in the following quote from a patient who was already under the care of the NHS for progressive low back pain 'I went to sleep on the Monday evening, woke up the Tuesday morning with obviously what I know now as the saddle numbness and not knowing that saddle numbness was part of the initial coming on of the Cauda Equina I just carried on again'. One of the additional challenges faced by patients is that some of the symptoms of CES may be considered embarrassing and there could be reluctance by some people to share these with clinicians Lavy et al. (2009).
We followed the model outlined by Vrkljan et al. (2010) who developed a Toolkit using the experience of both patients and clinicians. Similarly, the development of this CES toolkit drew on experiences of CES patients and experienced clinicians working in this field. Toolkits have been shown to be a cost effective method of disseminating practical evidence based guidelines to a wide audience (Shah et al., 2010). Eraut (2000) suggested that checklists such as the one developed on the clinician's cue card can assist in developing 'routinisation of clinical action' which will lead to improved patient outcomes. We anticipate that the CES toolkit will support health professionals from a range of disciplines in tailoring their evidence based care to the needs of the patient (Chatterjee, 2012).

\section{Strengths and weaknesses}

Strengths of the study are that the patients had direct lived experience of CES and the therapists were all experienced in managing potential CES sufferers. The main limitations are related to the small sample size and relatively small geographical distribution of the participants which may limit generalizability.

\section{Conclusions}

The findings of this research have helped to establish clear, sensitive and understandable guidelines for clinical questioning surrounding potentially embarrassing but critical symptoms. Synthesising the data generated from patient narratives with existing CES literature we have constructed a three-arm toolkit to use with patients considered by the clinician as at risk of developing CES (eg, worsening low back pain with symptoms/ signs of progressive sensory-motor deficit in the lower limbs). The three arms are Patient Expertise; Clinical Expertise; Research and Pathways. Uniquely the toolkit drew upon the lived experiences of patients suffering from CES to inform the content, helping to ensure that this study has produced results that are important to the public (NIHR, 2014).

Further validation is now required with a wider range of stakeholder groups such as novice physiotherapists and other relevant clinical specialities across Primary and Secondary care settings. We are 
currently seeking RfPB funding for a project to assess the feasibility of large scale use of the toolkit.

\section{Acknowledgements}

We would like to thank all the patients who generously gave up their time for this project and the Physiotherapy Research Foundation for funding this study.

\section{Funding}

This study was kindly funded by the Chartered Society of Physiotherapy Research Fund; PRF Reference Number; PRF 11/06. The sponsors had no involvement in the study itself.

\section{Conflicts of Interest}

There are no conflicts of interest.

\section{References}

André, M., Andén, A., Borgquist, L. and Rudebeck, C. 2012: GPs' decision-making - perceiving the patient as a person or a disease. BMC Family Practice 13, 38.

Anthony, S. 2003: Cauda equina syndrome. UK Casebook 20, 9-13.

Bin, M., Hong, W., Lian-shun, J., Wen, J., Guo-dong, S. and Jian-gang, S. 2009: Cauda equina syndrome: a review of clinical progress. Chinese Medical Journal 122, 1214-222.

Buchanan, E. 2013: How commonly do patients self-report subjective symptoms of cauda equina syndrome when asked on a screening form in a musculoskeletal triage service. Poster presentation, CSP Congress, Birmingham.

Chau, A.M., Xu, I.l., Pelzer, N.R. and Gragnaniello, C. 2014: Timing of surgical intervention in cauda equina syndrome: a systematic review. World Neurosurge 81, 640-50.

Cauda Equina Charity UK. 2014: (official website). Retrieved 3 February 2014 from http://www. 432caudaequinauk.com/ welcome.php

Chatterjee, J. 2012: Improving pain assessment for patients with cognitive impairment: development of a pain assessment toolkit. International Journal of Palliative Nursing 18/12, 581-90.

Eraut, M. 2000: Non-formal learning and tacit knowledge in professional work. British Journal of Educational Psychology 70, 113-36.

Fairbank, J. 2014: Cauda Equina Syndrome - risk management. Journal of Trauma and Orthopaedics 2, 49-50.

Fraser, S., Roberts, L. and Murphy, E. 2009: Cauda equina syndrome. A literature review of its definition and clinical

Primary Health Care Research \& Development 2016; 17: 559-567 presentation. Archives of Physical Medicine and Rehabilitation 90, 1964-968.

${ }^{\circledR}$ GHNHSFT. 2013: Physiotherapy guideline cauda equina syndrome (CES) - early Recognition v2 ISSUE DATE: v1 July 2012, v2 Apr 2013. Retrieved 14 November 2013 from: www.esp-physio.co.uk

Gleave, J.R.W. and Macfarlane, R. 2002: Cauda equina syndrome: what is the relationship between timing of surgery and outcome? British Journal of Neurosurgery 16, 325-28.

Greenhalgh, S., Truman, C., Webster, V. and Selfe, J. 2014: An investigation into the patient experience of cauda equina syndrome. Poster presentation, CSP Congress, Liverpool.

Greenhalgh, S., Truman, C., Webster, V. and Selfe, J. 2015: An investigation into the patient experience of cauda equina syndrome (CES). Physiotherapy Practice and Research 36, 23-31.

Jalloh, I. and Minhas, P. 2007: Delays in the treatment of cauda equina syndrome due to its variable clinical features in patients presenting to the emergency department. Emergency Medicine Journal 24, 33-34.

Korse, N., Jacobs, W., Elzevier, H. and Vleggeert- Lankamp, C. 2013: Complaints of micturition, defecation and sexual function in cauda equina syndrome due to lumbar disk herniation: a systematic review. European Spine Journal 22, 1019-29.

Lavy, C., James, A., Wilson-MacDonald, J. and Fairbank, J. 2009: Cauda equina syndrome. British Medical Journal 338, 881-84.

Markham, D.E. 2004: Cauda equina syndrome: diagnosis, delay and litigation risk. Journal of Orthopaedic Medicine 26, 102-5.

Mestrum, R., De Vooght, P., Vanelderen, P., Puylaert, M., Hans, G., Heylen, R., Vercauteren, M. and Van Zundert, J. 2009: Cauda equina syndrome secondary to lumbar disc herniation: pitfalls in clinical pain management. European Journal of Pain 13, S138-139.

Mitchell, E., Rubin, G. and Macleod, U. 2012: Improving diagnosis of cancer: a toolkit for general practice. Retrieved 16 January 2015 from www.rcgp.org.uk/.../Cancer/Improv ing \% 20Cancer \%20Diagnosis \% 20-\%20A \%20Toolkit \% 20 for \%20General\%20Practice \%20(2).ashx

Monroe, M. 2000: The value of a toolkit. Retrieved 9 February 2015 from http://www.joe.org/joe/2000december/tt5.php

NHS Litigation Authority. 2013: Freedom of Information request CES UK Charity.

NIHR. 2014: Involve website. Retrieved 31 March 2014 from http://www.invo.org.uk/

Selius, B. and Subedi, R. 2008: Urinary retention in adults: diagnosis and management. American Family Physician 77, 643-50.

Shah, B.R., Bhattacharyya, O., Yu, C., Mamdani, M., Parsons, J.A., Straus, S.E. and Zwarenstein, M. 2010: Evaluation of a toolkit to improve cardiovascular disease screening and treatment for people with type 2 diabetes: protocol for a cluster-randomized pragmatic trial. Trials [Electronic Resource] 11, 1745-6215. 
Sun, J.C., Xu, T., Chen, K.F., Qian, W., Liu, K., Shi, J.G., Yuan, W. and Jia, L.S. 2014: Assessment of cauda equina syndrome progression pattern to improve diagnosis. Spine $39,596-602$.

Turnpenney, J., Greenhalgh, S., Richards, L., Crabtree, A. and Selfe, J. 2013: Developing an early alert system for metastatic spinal cord compression. Primary Health Care Research \& Development 16, 14-20.

Underwood, M. 2009: Diagnosing acute nonspecific low back pain: time to lower the red flags? Arthritis \& Rheumatology $60,2855-857$.
Vrkljan, B.H., Cranney, A., Worswick, J., O'Donnell, S., Li, L.C., Gelinas, I., Byszewski, A., Man-Son-Hing, M. and Marshall, S. 2010: Supporting safe driving with arthritis: developing a driving toolkit for clinical practice and consumer use. American Journal of Occupational Therapy 64, 259-67.

Woolsley, R. and Martin, D. 2003: Spinal cord and cauda equina syndromes. In Lin V., Cardenas D., Cutter N. et al, editors Spinal cord medicine: principles and practice. New York: Demos Medical Publishing New York, 407-27. 